Chapter 9, Ocular Surface Immune Reactions and Contact Lens Wear, has only two references from the 1990s and the more recent of these is 1993 . It contains nothing that is not common knowledge amongst contact lens practitioners.

Chapter 10, Keratitis and Contact Lenses, is a brief but useful overview of the present thoughts and state of the art in the field of disposable lenses. Its critical and economical style makes it a pleasure to read. I am suspicious that Figure 10.2 is of a corneal nerve and not Aspergillus fumigatus. The chapter ends with the not surprising conclusion that daily-disposable contact lenses when worn as prescribed are safest.

I was left wondering why this book was assembled, what it was intended to do and for what audience.

P.N. Dilly

\section{The Eye in General Practice}

R.D. Finlay and P.A.G. Payne Heinemann, 1997

An interesting little book this, with much to commend it to the doctor who wishes to have a quick and comprehensive overview of common ocular problems, particularly if there is little desire to know what the specialist might do to treat a particular condition. I think it might be an excellent primer for a student or a very good basic background text for a GP registrar. Unfortunately I do not think it begins in any way to live up to its title. I think more appropriately termed 'Ophthalmic Problems for the Uninitiated' it would do very well.

Sadly the book starts badly, suggesting as it does that an essential tool for the examination of the eye in general practice is a slit lamp. I performed a straw poll of a number of GP colleagues and was able to ascertain that, although many actually knew that a slit lamp was a tool used by an ophthalmologist, none could with any certainty identify where they would expect to find the on/off switch. Not surprisingly I was also unable to find a GP who owned a slit lamp. We really can do without books for general practice which clearly demonstrate such a lack of understanding of the role of the GP in managing ophthalmic conditions.

General practitioners, on the other hand, have a very different agenda in their management of eye disorders in their patients. Put simply they require to know what they may safely diagnose and treat and what they must refer and what they must not attempt to treat. All the information required to perform these functions is, I am sure, available within the pages of this book, it is just that, organised as it is, the material is inaccessible to the GP rapidly.

An example of this is the series of tables which appears in Chapter 2. Table 2.1 is titled 'The Acute Red Eye' and contains a series of causes of the acute red eye with a clinical description

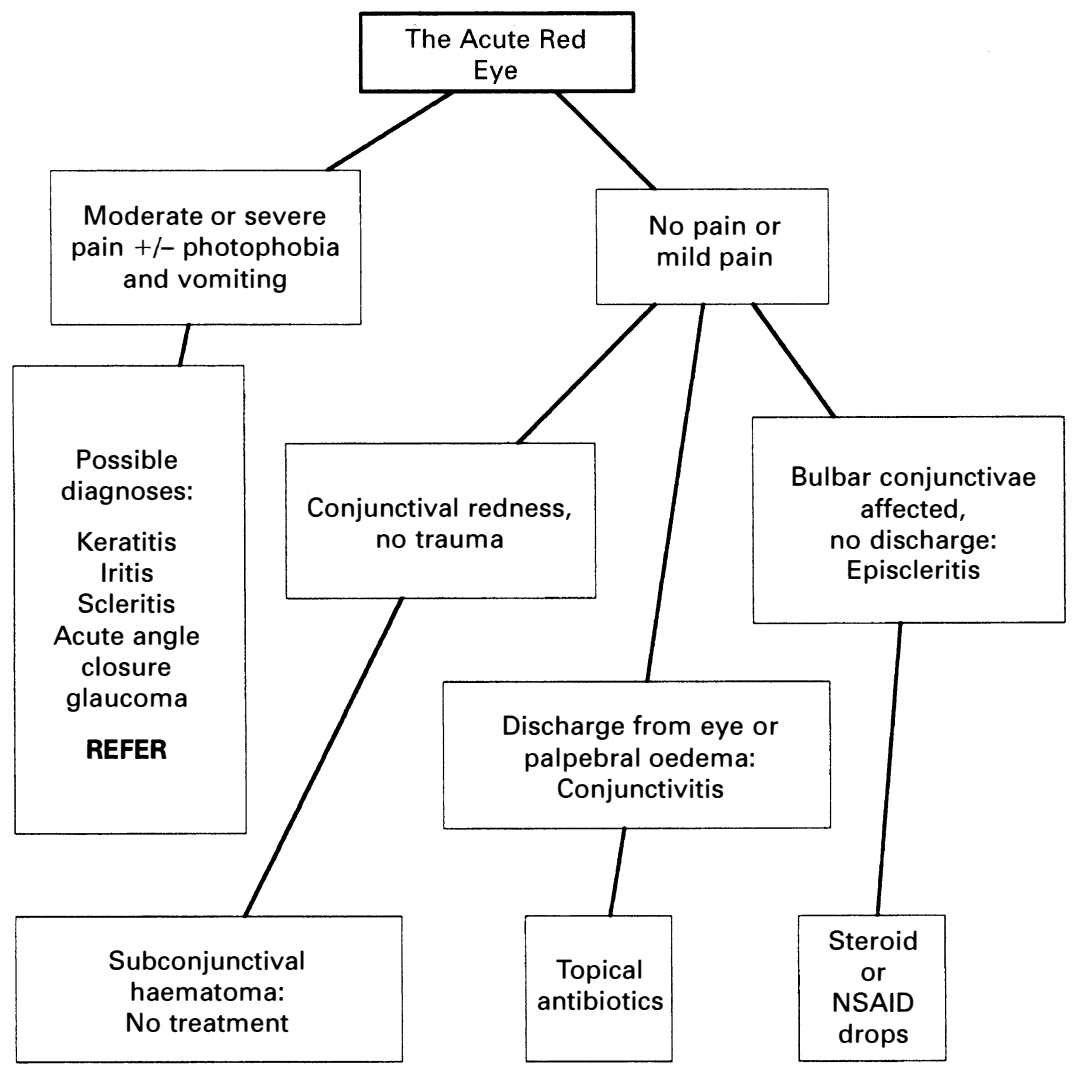

Fig. 1. followed by a treatment. Four of the conditions described have a suggested treatment of 'refer'. Surely a more appropriate vade-mecum of GP ophthalmological problems would use a decision tree such as that in Fig. 1 to inform the GP of appropriate diagnosis and/or disposal.

Thus this very good little book could be excellent if its content were reorganised to be useful to its target audience. I think that a small primer which gave a sufficiently easy to use series of diagnostic algorithms would rapidly gain acceptance as a GP standard text - a position which I fear this book is not destined to occupy. John Poyser

Ophthalmology - Colour Guide,
2nd edition

Jack J. Kanski

Churchill Livingstone, 1997

\section{Pocket Book of Ophthalmology}

Philip I. Murray and Alistair R. Fielder Butterworth-Heinemann, 1997

'. . . vether it's worth while goin' through so much to learn so little, as the charity boy said ven he got to the end of the alphabet, is a matter of taste', said Mr Weller in Pickwick Papers. For those without the time or money to invest in the third edition of Clinical Ophthalmology, JJK's Colour Guide is the 'charity boy' edition.

It would be a breach of hippocratic dogma for any British-trained ophthalmologist of my generation to have anything negative to say about any book by JJK. This volume is a pocketsized and slimline condensation of the great tome on which we were all raised. It contains many of the same beautiful illustrations with a pithy bullet-form commentary accompanying. The quality of the illustrations is uniformly excellent (e.g. Vogt's striae on p. 38). It will, no doubt, be popular among medical students and brand-new SHOs, for whom the visual impact and verbal brevity will be very appealing, but it has little to offer anyone who will have to invest in the larger volume for their future training.

Aimed at much the same audience, Murray and Fielder's book is the same size and has not one photograph in it, which will make it hard to sell. It is a book of lists, despite the disclaimer in the introduction. It is likely to be perceived as too specialist by the generalist (who wants to see pictures) and inadequate by the specialist trainee (who will have invested in a well-known and more encyclopaedic book of ophthalmic lists - also without pictures). It is not consistent in the level and pitch of the script, in which it is felt necessary to explain the word 'stye' ('infection of eye lash root') but leaves 'oscillopsia' 